\title{
Spectral characterization of a graph on the flags of the eleven point biplane
}

\author{
A. Blokhuis - A. E. Brouwer
}

Received: 22 June 2011 / Revised: 17 September 2011 / Accepted: 19 September 2011 /

Published online: 2 October 2011

(C) The Author(s) 2011. This article is published with open access at Springerlink.com

\begin{abstract}
We characterize a 55-point graph by its spectrum $4^{1},(-2)^{10},(-1 \pm \sqrt{3})^{10}$, $((3 \pm \sqrt{5}) / 2)^{12}$. No interlacing is used: examination of $\operatorname{tr} A^{m}$ for $m \leq 7$ together with study of the representation in the eigenspace for the eigenvalue -2 suffices.
\end{abstract}

Keywords Spectral characterization - Euclidean representation - Graph representation · Iofinova-Ivanov graph

\section{Mathematics Subject Classification (2000) $\quad 05 \mathrm{C} 40 \cdot 05 \mathrm{Bxx} \cdot 05 \mathrm{Exx} \cdot 05 \mathrm{C} 38$}

\section{A graph with few cycles}

Up to isomorphism, there is a unique symmetric 2-(11,5,2) design, given for example by the 11 elements of the field $\mathbb{F}_{11}$ (as points) together with the 11 translates of the set $\{1,3,4,5,9\}$ of nonzero squares (as blocks). The design condition means that any pair of points occurs in precisely 2 blocks, and, dually, any two blocks meet in precisely two points. Such designs are known as biplanes. A flag is an incident point-block pair.

Fix a 2-(11,5,2) biplane, and construct a graph $\Gamma$ on the 55 flags by defining $(x, B) \sim$ $(y, C)$ iff $B \cap C=\{x, y\}$. The distance distribution diagram of $\Gamma$ is

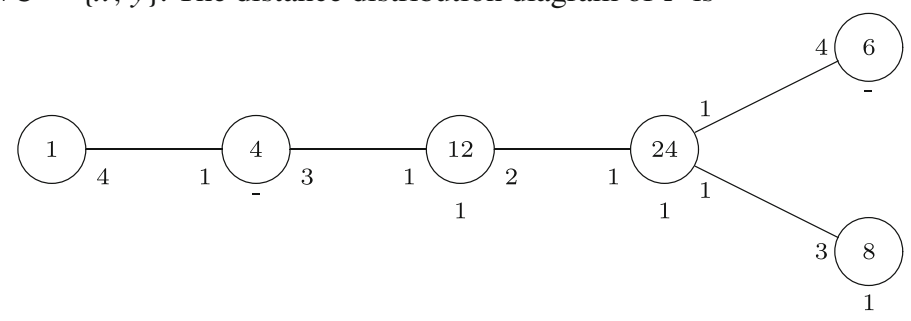

This is one of several papers published together in Designs, Codes and Cryptography on the special topic: "Geometric and Algebraic Combinatorics".

A. Blokhuis · A. E. Brouwer $(\varangle)$

Eindhoven University of Technology, Eindhoven, The Netherlands

e-mail: aeb@cwi.nl 
This graph has pentagons, but no other induced cycles of length less than 8 . The spectrum of $\Gamma$ is $4^{1},(-2)^{10},(-1 \pm \sqrt{3})^{10},((3 \pm \sqrt{5}) / 2)^{12}$. We shall investigate graphs with the same spectrum.

\section{Cycles}

Let now $\Gamma$ be a graph with adjacency matrix $A$ with spectrum $4^{1},(-2)^{10},(-1 \pm \sqrt{3})^{10},((3 \pm$ $\sqrt{5}) / 2)^{12}$. Write $d(x, y)$ for the graph distance of the vertices $x$ and $y$ in $\Gamma$. Write $\Gamma_{i}(x):=$ $\{y \mid d(x, y)=i\}$. We show that $\Gamma$ has the above diagram around each of its vertices.

First of all, $\Gamma$ has 55 vertices, since there are 55 eigenvalues. The number of edges is $\frac{1}{2} \operatorname{tr} A^{2}=110$ so that the average valency is 4 , but 4 is the largest eigenvalue, hence $\Gamma$ is regular of valency 4. Since the eigenvalue 4 has multiplicity $1, \Gamma$ is connected. Since $\operatorname{tr} A^{3}=0$, there are no triangles.

We have $\operatorname{tr} A^{4}=1540$. The contribution of trivial walks of length 4 ( $x-y-x-z-x$ and $x-y-z-y-x)$ is $55 \cdot(4 \cdot 4+4 \cdot 3)=1540$, so this is all, and there are no induced quadrangles.

We have $\operatorname{tr} A^{6}=12760$. The contribution of trivial walks of length $6(x-y-x-z-x-w-x$ and $x-y-x-z-w-z-x$ and $x-y-z-y-x-w-x$ and $x-y-z-y-w-y-x$ and $x-y-z-w-z-y-x)$ is $55 \cdot(4 \cdot 4 \cdot 4+$ $2 \cdot 4 \cdot 4 \cdot 3+4 \cdot 3 \cdot 3+4 \cdot 3 \cdot 3)=12760$, so this is all, and there are no induced hexagons.

We have $\operatorname{tr} A^{5}=660=55 \cdot 4 \cdot 3$ so that the average number of pentagons on a 2 -claw (path of length 2) is 1 . But if two pentagons have two consecutive edges in common, we see an induced hexagon, which is impossible. So, every 2-claw lies in exactly one pentagon, and every vertex lies in 6 pentagons.

We have $\operatorname{tr} A^{7}=13860$. The contribution of the walks of length 7 that consist of a pentagon plus an edge traversed in both directions equals $55 \cdot(4 \cdot 12+12 \cdot 5 \cdot 3+4 \cdot 6)=13860$, so this is all, and there are no induced heptagons. It follows that if $v w$ is an edge in $\Gamma_{3}(x)$, then the only walk of length 7 from $x$ via $v w$ back to $x$ is $x-y-z-w-v-u-y-x$ and $v$ is the unique neighbour of $w$ in $\Gamma_{3}(x)$ (since the pentagon $y-z-w-v-u-y$ is determined by $w-z-y$ ).

This gives most of the diagram. The final bits will be seen below.

\section{10-Dimensional representation}

Consider the matrix $M=2 J-\left(A^{2}+2 A-2 I\right)\left(A^{2}-3 A+I\right)$. Since $A J=J A$, eigenvectors of $A$ are also eigenvectors of $M$, and we find that $M$ has spectrum $0^{45}, 22^{10}$, hence is positive semi-definite of rank 10 . Write $M=N^{\top} N$ for a real $10 \times 55$ matrix $N$. The map $x \mapsto \bar{x}$ that sends $x$ to the column of $N$ indexed by $x$ is a representation of $\Gamma$ in $\mathbb{R}^{10}$. We shall show that

$$
(\bar{x}, \bar{y})=\left\{\begin{array}{cc}
4 & \text { if } d(x, y)=0 \\
-2 & \text { if } d(x, y)=1 \\
0 & \text { if } d(x, y)=2 \\
1 & \text { if } d(x, y)=3 \\
2-c_{4} & \text { if } d(x, y) \geq 4
\end{array}\right.
$$

where $c_{4}=c_{4}(x, y)$ (for $d(x, y) \geq 4$ ) is the number of neighbours of $y$ that have distance 3 to $x$.

Let $A_{i}$ be the matrix with rows and columns indexed by the vertex set of $\Gamma$, with $\left(A_{i}\right)_{x y}=1$ when $d(x, y)=i$, and $\left(A_{i}\right)_{x y}=0$ otherwise. From the (known part of) the diagram we see 
that $A_{2}=A^{2}-4 I$ and $A_{3}=A A_{2}-3 A-A_{2}$, so that $M=2 J-\left(A_{2}+2 A+2 I\right)\left(A_{2}-\right.$ $3 A+5 I)=2 J+2 I-4 A-A A_{3}$ from which the claim follows.

So far we have no use for this observation, but if $a, b, c, d$ are the four neighbours of $x$, then $\|2 \bar{x}+\bar{a}+\bar{b}+\bar{c}+\bar{d}\|^{2}=16-16=0$, so that $2 \bar{x}+\bar{a}+\bar{b}+\bar{c}+\bar{d}=0$.

\section{Pentagons}

The graph $\Gamma$ has 66 pentagons, 6 on each vertex. If $x-y-z-w-v-x$ is a pentagon, then $\| \bar{x}+$ $\bar{y}+\bar{z}+\bar{w}+\bar{v} \|^{2}=20-20=0$ so that $\bar{x}+\bar{y}+\bar{z}+\bar{w}+\bar{v}=0$.

Fix a vertex $a$, and look at the distributions of distances from $a$ to the five vertices of each pentagon. We claim that these are as follows.

\begin{tabular}{llllllll}
\hline$(\bar{a}, \bar{x})$ & 4 & -2 & 0 & 1 & -2 & -1 & \\
$d(a, x)$ & 0 & 1 & 2 & 3 & $4 \mathrm{a}$ & $4 \mathrm{~b}$ & $\#$ \\
\hline & 1 & 2 & 2 & 0 & 0 & 0 & 6 \\
& 0 & 1 & 2 & 2 & 0 & 0 & 12 \\
& 0 & 0 & 2 & 2 & 1 & 0 & 12 \\
& 0 & 0 & 1 & 2 & 0 & 2 & 12 \\
& 0 & 0 & 0 & 3 & 1 & 1 & 24 \\
\hline
\end{tabular}

Of course, for a pentagon $\left\{x_{1}, x_{2}, x_{3}, x_{4}, x_{5}\right\}$ we must have $\sum_{i=1}^{5}\left(\bar{a}, \overline{x_{i}}\right)=0$. Also recall that each 2-claw is in a unique pentagon.

The first two rows are clear and cover all pentagons on $a$ or a neighbour of $a$. Consider a pentagon with distance at least 2 to $a$. With an edge in $\Gamma_{2}(a)$ the distribution must be as given by the third row, with a vertex $z$ in $\Gamma_{4}(a)$ for which $(\bar{a}, \bar{z})=-2$, i.e., $c_{4}(a, z)=4$. With a single vertex in $\Gamma_{2}(a)$ we cannot have an edge in $\Gamma_{3}(a)$ (since that would force a distribution as in the second row), so have a distribution as in the fourth row, with an edge $y z$ in $\Gamma_{4}(a)$, where $c_{4}(a, y)+c_{4}(a, z)=6$. But $c_{4}(a, y), c_{4}(a, z) \leq 3$ since $y, z$ have a neighbour in $\Gamma_{4}(a)$, so $c_{4}(a, y)=c_{4}(a, z)=3$ in this case. This shows that each vertex in $\Gamma_{3}(a)$ has two neighbours $y, z$ in $\Gamma_{4}(a)$, one with $c_{4}(a, y)=3$ and one with $c_{4}(a, z)=4$. This completes the proof for the distribution diagram, and also shows that the remaining pentagons are described by the last row of the above table.

We shall use $\Gamma_{4 a}(x)$ and $\Gamma_{4 b}(x)$ for the sets of vertices at distance 4 from $x$ in relation $4 \mathrm{a}$ or $4 \mathrm{~b}$, respectively.

\section{Labels}

We show that $\Gamma$ can be described as follows. The vertices are labeled $\infty, i, i j, i j k, i j k l, i j k *$ with $i, j, k, l$ distinct elements of $I=\{1,2,3,4\}$, where the four labels $i j k l, j i l k, k l j i, l k i j$ denote the same vertex and the three labels $j i k *, k i l *, l i j *$ denote the same vertex (for all sets of pairwise distinct $i, j, k, l \in I$ ), so that the number of vertices is $1+4+12+24+$ $24 / 4+24 / 3=55$.

The adjacencies are given by

\begin{tabular}{lcccccc}
\hline vertex & $\infty$ & $i$ & $i j$ & $i j k$ & $i j k l$ & $i j k *$ \\
\hline neighbours & $i$ & $\infty, i j$ & $i, j i, i j k$ & $i j, i k j, i j k l, i j k *$ & $i j k$ & $i j k, i j l *$ \\
\hline
\end{tabular}

That this labeling and these adjacencies are forced uniquely will show that $\Gamma$ is determined by its spectrum. 
Fix a vertex and label it $\infty$. Label its four neighbours $i$ for $i \in I$. From the diagram, and the known pentagons we now see that all vertices can be labeled uniquely as described, with all adjacencies as described. The only thing left to show is which sets of three or four labels belong to the same vertex in $\Gamma_{4}(\infty)$.

We need some more detailed information first, and collect the relation of all vertices to the vertices $\infty$ and $i$.

(i) With respect to $\infty$ we have, by definition,

\begin{tabular}{lllllll}
\hline & $\infty$ & $i$ & $i j$ & $i j k$ & $i j k l$ & $i j k *$ \\
\hline dist & 0 & 1 & 2 & 3 & $4 \mathrm{a}$ & $4 \mathrm{~b}$ \\
inprod & 4 & -2 & 0 & 1 & -2 & -1 \\
\hline
\end{tabular}

where for a vertex $x$ 'dist' denotes $d(\infty, x)$ and 'inprod' denotes $(\bar{\infty}, \bar{x})$.

(ii) With respect to 1 we have (with 2, 3, 4 still equivalent)

\begin{tabular}{lllllllllll}
\hline & $\infty$ & 1 & 2 & 12 & 21 & 23 & 123 & 213 & 231 & 234 \\
\hline dist & 1 & 0 & 2 & 1 & 2 & 3 & 2 & 3 & $4 \mathrm{a}$ & $4 \mathrm{~b}$ \\
inprod & -2 & 4 & 0 & -2 & 0 & 1 & 0 & 1 & -2 & -1 \\
\hline & $i j k l$ & $123 *$ & $213 *$ & $231 *$ & $234 *$ & & & & & \\
\cline { 1 - 5 } dist & 3 & 3 & $4 \mathrm{~b}$ & 3 & 3 & & & & & \\
inprod & 1 & 1 & -1 & 1 & 1 & & & & & \\
\hline
\end{tabular}

Indeed, the path $1 \sim 12 \sim 21 \sim 213$ shows that $d(1,213)$ is at most 3 , but $d(1,213)$ is not 2 , since the unique pentagon on $1 \sim 12 \sim 21$ is $\infty \sim 1 \sim 12 \sim 21 \sim 2 \sim \infty$.

The pentagon $2 \sim 21 \sim 213 \sim 231 \sim 23 \sim 2$ has inprods summing to zero, and we already see $0+0+1+1$, so that 231 must contribute -2 .

Since $d(\infty, 123)=3$, the distances $d(1,123), d(2,123), d(3,123), d(4,123)$ must be $2,3,4 \mathrm{a}, 4 \mathrm{~b}$ in some order, and therefore in this order. This shows that $d(1,234)=4 b$.

Since the relation $4 \mathrm{a}$ is symmetric (it is equivalent to having distance 4 where the representing vectors have inner product -2 ), $\infty$ has relation 4 a to each $i j k l$, so its neighbour 1 has distance 3 to $i j k l$.

Since $d(1,213)=3$, the distances $d(1,21), d(1,2134), d(1,231), d(1,213 *)$ must be 2 , $3,4 \mathrm{a}, 4 \mathrm{~b}$ in some order, and therefore in this order. Now $\infty$ and 1 have relation $4 \mathrm{~b}$ to $213 *$, while the other neighbours 2, 3, 4 of $\infty$ have relation 3 to $213 *$. We see that the three labels of $i j k *$ start with $i, k, l$ but not with $j$.

That settles the above table. Now back to the labels.

We already saw that a vertex $x \in \Gamma_{4 a}(\infty)$ has four labels, starting with four distinct symbols. The pentagon $i j \sim i j k \sim i j k l \sim j i$ ? $\sim j i \sim i j$ shows that if $x$ has label $i j k l$, then also $j i$ ??. For $i j k l=2314$, the inner products of the representing vectors of the vertices of this pentagon with $\overline{1}$ must add up to zero. We see (omitting the bars) $(1,23)=(1,32)=$ $(1,2314)=1,(1,231)=-2$, so that the fifth inner product must be -1 and the fifth vertex 32 ? was 324 . It follows that 2314 and 3241 label the same vertex.

We have shown that the four labels of 1234 are 1234, 2143, 34??, 43??. These last two labels will be 3421 and 4312 if we show that 1234 is distinct from 3412. Make a table with relations and inner products with respect to 13 .

\begin{tabular}{lllllllll}
\hline & 12 & 21 & 34 & 43 & 123 & 214 & 341 & 432 \\
\hline dist & 2 & 3 & 3 & $4 \mathrm{a}$ & 2 & $4 \mathrm{a}$ & 3 & 3 \\
inprod & 0 & 1 & 1 & -2 & 0 & -2 & 1 & 1 \\
\hline
\end{tabular}


(For 43, look at the pentagon $\infty \sim 3 \sim 34 \sim 43 \sim 4 \sim \infty$ and take inner products with 13. Since $d(13,43)=4 a$ and $43 \sim 432$, we have $d(13,432)=3$. For 214 look at the pentagon $\infty \sim 1 \sim 13 \sim 31 \sim 3 \sim \infty$ and take inner products with 214.)

Using the pentagons $12 \sim 123 \sim 1234=2143 \sim 214 \sim 21 \sim 12$ and $34 \sim 341 \sim$ $3412=4321 \sim 432 \sim 43 \sim 34$, we see that $(13,1234)=1$ and $(13,3412)=-1$, so that 1234 and 3412 label different vertices.

That settles the labeling of $\Gamma_{4 a}(\infty)$. Next $\Gamma_{4 b}(\infty)$. We already know that each vertex has three labels, with three different first elements, and the same second element, so that $123^{*}$, 32 ? $^{*}, 42$ ? $^{*}$ label the same vertex. These last two labels will be $324^{*}$ and $421^{*}$ if we show that $123 *$ is distinct from $321 *$.

Look at the pentagon $12 \sim 123 \sim 123 * \sim 124 * \sim 124 \sim 12$. Since $d(13,12)=$ $d(13,123)=2$, we must have $d(13,124)=d(13,123 *)=3$ and $d(13,124 *)=4 a$. Look at the pentagon $32 \sim 321 \sim 321 * \sim 324 * \sim 324 \sim 32$. Since $d(13,32)=d(13,321)=3$ we have $d(13,321 *) \neq 3$. This proves everything.

We proved the following result.

Theorem 5.1 There is a unique graph $\Gamma$ with spectrum $4^{1},(-2)^{10},(-1 \pm \sqrt{3})^{10},((3 \pm$ $\sqrt{5}) / 2)^{12}$.

\section{Remarks}

The graph $\Gamma$ has automorphism group $L_{2}(11) .2$ with point stabilizer Sym(4).

Related graphs occur in the literature. The graph $\Gamma_{4 a}$ of valency 6 has $\lambda=1$ and hence is the collinearity graph of a partial linear space with 55 points and 55 lines. The point-line incidence graph $\Delta$ of this partial linear space is known as the Iofinova-Ivanov graph on 110 vertices. It was first constructed in Ivanov [1] and characterized by Ivanov and Iofinova [2] as one of the five connected bipartite cubic graphs with an automorphism group that is edge-transitive but not point-transitive, acting primitively on the two point orbits.

It follows that $\Gamma_{4 a}$ is not characterized by its spectrum: the two connected components of $\Delta_{2}$ are cospectral but nonisomorphic.

Open Access This article is distributed under the terms of the Creative Commons Attribution Noncommercial License which permits any noncommercial use, distribution, and reproduction in any medium, provided the original author(s) and source are credited.

\section{References}

1. Ivanov A.A.: Computations of lengths of orbits of a subgroup in a transitive permutation group. In: Faradžev I.A., Ivanov A.A., Klin M.H., Woldar A.J. (eds.) Methods of Complex Systems Study, pp. 3-8. Institute for System Studies, Moscow (1983) (Russian) [Translation in Investigations in Algebraic Theory of Combinatorial Objects, pp. 275-282. Kluwer, Dordrecht (1994)].

2. Ivanov A.A., Iofinova M.E.: Bi-primitive cubic graphs. In: Investigations in the Algebraic Theory of Combinatorial Objects, pp. 123-134. Institute for System Studies, Moscow (1985) (Russian) [Translation pp. 459-472, Kluwer, Dordrecht (1994)]. 